\title{
Submanifolds of Sasakian Manifolds with Concurrent Vector Field
}

\author{
Pradip Mandal, Yadab Chandra Mandal and Shyamal Kumar Hui
}

\begin{abstract}
The submanifolds of Sasakian manifolds with a concurrent vector field have been studied. Applications of such submanifolds to Ricci solitons and Yamabe solitons has also been showed.
\end{abstract}

\section{Introduction}

Sasakian manifold $\bar{M}$ is a $(2 n+1)$-dimensional almost contact metric manifold such that [1]

$$
\begin{aligned}
\left(\bar{\nabla}_{X} \phi\right) Y & =g(X, Y) \xi-\eta(Y) X \\
\bar{\nabla}_{X} \xi & =-\phi X
\end{aligned}
$$

where $(\phi, \xi, \eta, g)$ is the almost contact metric structure and $\bar{\nabla}$ is the Riemannian connection on $\bar{M}$. A vector field $X$ on $\bar{M}$ is said to be conformal if

$$
\mathcal{L}_{X} g=2 \alpha g
$$

where $\alpha \in C^{\infty}(\bar{M})$ and $\mathcal{L}_{X}$ denotes the Lie derivative along $X$. In particular, if $\alpha=0$ then $X$ is Killing. And $X$ is said to be concurrent if

$$
\bar{\nabla}_{Z} X=Z
$$

for any $Z \in \chi(\bar{M})$.

Let $M$ be an $m$-dimensional submanifold of $\bar{M}$. A Ricci soliton on $M$ is a triplet $(g, W, \sigma)$ such that [12]

$$
\mathcal{L}_{W} g+2 S+2 \sigma g=0
$$

2010 Mathematics Subject Classification. 53C42, 53C43.

Key words and phrases. Submanifold, Sasakian manifold, concurrent vector field, conformal vector field, Ricci soliton, Yamabe soliton.

Corresponding author: Shyamal Kumar Hui. 
where $S$ is the Ricci tensor on $M, W$ is the potential vector field and $\sigma \in \mathbb{R}$. An Yamabe soliton on $M$ is a triplet $(g, W, \lambda)$ such that

$$
\frac{1}{2} \mathcal{L}_{W} g=(r-\lambda) g,
$$

where $r$ is the scalar curvature on $M$ and $\lambda \in \mathbb{R}$. If the dimension of $M$ is 2 then the notions of Ricci soliton and Yamabe soliton are equivalent. However, when the dimension of $M$ is greater than 2 , they are different.

Chen and his co-author studied Euclidean submanifold whose canonical vector field are concurrent [4], concircular [11], conformal [10], torse-forming [9] and also in ([3], [5], [6]). Ricci soliton and Yamabe soliton whose canonical vector field are concurrent and conformal studied in ([2], [7], [8]).

The object of the present paper is to study of submanifolds of Sasakian manifolds with concurrent vector field. We also apply such submanifolds to Ricci solitons and Yamabe solitons.

\section{Preliminaries}

An odd dimensional smooth manifold $\bar{M}^{2 n+1}$ is said to be an almost contact metric manifold if the following relations hold: [1]

$$
\begin{gathered}
\phi^{2} X=-X+\eta(X) \xi, \phi \xi=0, \\
g(X, \xi)=\eta(X), \phi \circ \eta=0, \\
g(\phi X, \phi Y)=g(X, Y)-\eta(X) \eta(Y)
\end{gathered}
$$

for all $X, Y \in \chi(\bar{M})$, where $\phi$ is a tensor of type $(1,1), \xi$ is a vector field, $\eta$ is an 1 -form and $g$ is a Riemannian metric on $\bar{M}$.

Let $\nabla$ and $\nabla^{\perp}$ be the induced connections on the tangent bundle $T M$ and the normal bundle $T^{\perp} M$ of $M$, respectively. Then we have

$$
\begin{aligned}
& \bar{\nabla}_{X} Y=\nabla_{X} Y+h(X, Y), \\
& \bar{\nabla}_{X} V=-A_{V} X+\nabla_{X}^{\perp} V,
\end{aligned}
$$

where $h$ and $A_{V}$ are second fundamental form and shape operator respectively for the immersion of $M$ into $\bar{M}$ and they are related by the following equation, see [13]

$$
g(h(X, Y), V)=g\left(A_{V} X, Y\right)
$$


for any $X, Y \in \Gamma(T M)$ and $V \in \Gamma\left(T^{\perp} M\right)$. If $h=0$, then $M$ is said to be totally geodesic.

Let $\left\{e_{i}: 1 \leq i \leq m\right\}$ be an orthonormal basis to the tangent space at any point of $M$. Then the mean curvature of $M$ is

$$
H=\frac{1}{m} \sum_{i=1}^{m} h\left(e_{i}, e_{i}\right) .
$$

And $M$ is said to be totally umbilical if

$$
h(X, Y)=g(X, Y) H
$$

Again $M$ is said to be umbilical with respect to $V \in T^{\perp} M$ if

$$
g(h(X, Y), V)=\mu g(X, Y)
$$

for some function $\mu$. In particular if $g(h(X, Y), H)=\mu g(X, Y)$ holds then $M$ is said to be pseudo-umbilical. Consider

$$
\phi X=P X+F X
$$

where $P X$ and $F X$ are the tangential and normal components of $\phi X$. And $M$ is called generalized self-similar submanifold of $\bar{M}$ if

$$
F X=f H
$$

where $f \in C^{\infty}(M)$.

\section{Results}

We now prove the followings:

Theorem 3.1. Let $M$ be a submanifold of $\bar{M}$ with a concurrent vector field $X$ such that $\xi$ is normal to $M$. Then $P X$ is conformal if and only if $M$ is umbilical with respect to $F X$.

Proof. Since $X$ is concurrent vector field of $\bar{M}$, we have from (1.4) that

$$
\begin{aligned}
\phi Z & =\phi \bar{\nabla}_{Z} X \\
& =\bar{\nabla}_{Z} \phi X-\left(\bar{\nabla}_{Z} \phi\right) X .
\end{aligned}
$$

Using (1.1), (2.4), (2.5) and (2.10) in (3.1) we have

$$
\begin{aligned}
P Z+F Z & =\bar{\nabla}_{Z}(P X+F X)-g(X, Z) \xi \\
& =\nabla_{Z} P X+h(Z, P X)+\nabla \frac{1}{Z} F X-A_{F X} Z-g(X, Z) \xi
\end{aligned}
$$


Comparing the tangential component of (3.2) we have

$$
\nabla_{Z} P X=P Z+A_{F X} Z
$$

Now we have

$$
\begin{aligned}
& \left(\mathcal{L}_{P X} g\right)(Y, Z) \\
& =g\left(\nabla_{Y} P X, Z\right)+g\left(Y, \nabla_{Z} P X\right) \\
& =g\left(P Y+A_{F X} Y, Z\right)+g\left(Y, P Z+A_{F X} Z\right) \\
& =g\left(A_{F X} Y, Z\right)+g\left(A_{F X} Z, Y\right) .
\end{aligned}
$$

Using (2.6) in (3.4) we have

$$
\left(\mathcal{L}_{P X} g\right)(Y, Z)=2 g(h(Y, Z), F X) .
$$

Suppose $P X$ is conformal. Then from (1.3) and (3.5) we have

$$
g(h(Y, Z), F X)=\alpha g(Y, Z),
$$

which implies that $M$ is umbilical with respect to $F X$.

Conversely, assume that $M$ is umbilical with respect to $F X$. Then from (2.9) and (3.5) we have

$$
\left(\mathcal{L}_{P X} g\right)(Y, Z)=2 \mu g(Y, Z)
$$

which means that $P X$ is conformal.

Theorem 3.2. Let $M$ be a submanifold of $\bar{M}$ with a concurrent vector field $X$. Then $X$ is a homothetic vector field.

Proof. Since $X$ is a concurrent vector field, so we have from (1.4) and (2.4) that

$$
\nabla_{Z} X+h(X, Z)=Z
$$

Equating tangential and normal components of (3.8) we get

$$
\nabla_{Z} X=Z, h(X, Z)=0 .
$$

Now we have

$$
\left(\mathcal{L}_{X} g\right)(Y, Z)=g\left(\nabla_{Y} X, Z\right)+g\left(Y, \nabla_{Z} X\right) .
$$

Using (3.9) in (3.10) we have

$$
\left(\mathcal{L}_{X} g\right)(Y, Z)=2 g(Y, Z),
$$

which implies that $X$ is conformal vector field of $M$ with constant function $\alpha=1$, i.e. $X$ is homothetic. 
Theorem 3.3. Let $M$ be a submanifold of $\bar{M}$ with a concurrent vector field $X$. If $(g, X, \sigma)$ is a Ricci soliton on $M$ then $M$ is Einstein and such a soliton is shrinking.

Proof. Since $(g, X, \sigma)$ is a Ricci soliton on $M$, we have the equation (1.5). Using (3.11) in (1.5) we get $S(Y, Z)=-(\sigma+1) g(Y, Z)$, which implies that $M$ is Einstein.

By virtue of (3.9) we get

$$
R(Y, Z) X=\nabla_{Y} \nabla_{Z} X-\nabla_{Z} \nabla_{Y} X-\nabla_{[Y, Z]} X=0,
$$

and hence $S(Y, X)=0$. So, $\sigma+1=0$, i.e., $\sigma=-1$. Hence the given Ricci soliton is shrinking.

Theorem 3.4. Let $M$ be a submanifold of $\bar{M}$ with a concurrent vector field $X$. If $(g, X, \lambda)$ is an Yamabe soliton on $M$ then such soliton is shrinking, steady and expanding according as $r<1, r=1$ and $r>1$ respectively.

Proof. Since $(g, X, \lambda)$ is an Yamabe soliton on $M$, we have the equation (1.6). Using (3.11) in (1.6) we get $\lambda=r-1$. Hence the result.

Theorem 3.5. Let $M$ be a submanifold of $\bar{M}$ with a concurrent vector field $X$ such that $\xi$ is normal to $M$. If $(g, P X, \lambda)$ is an Yamabe soliton on $M$, then $P X$ is conformal.

Proof. Let $(g, P X, \lambda)$ be an Yamabe soliton on $M$. Then from the equation (1.6), we get

$$
\frac{1}{2}\left(\mathcal{L}_{P X} g\right)(Y, Z)=(r-\lambda) g(Y, Z) .
$$

From (3.5) and (3.12) we have

$$
g(h(Y, Z), F X)=(r-\lambda) g(Y, Z)
$$

for all $Y, Z \in \Gamma(T M)$, which implies that $M$ is umbilical with respect to $F X$. Then by virtue of Theorem 3.1, it follows that $P X$ is conformal.

Theorem 3.6. Let $M$ be a generalized self-similar submanifold of $\bar{M}$ with a concurrent vector field $X$ such that $\xi$ is normal to $M$. Then $P X$ is conformal vector field if and only if $M$ is pseudo-umbilical.

Proof. Let $M$ be a generalized self-similar submanifold of $\bar{M}$, then we have the equation (2.11). If $P X$ is conformal vector field, then we have the equation (3.6). From (2.11) and (3.6) we can say that $M$ is pseudo-umbilical.

Conversely, if $M$ is pseudo umbilical submanifold then from equation (2.11) we say that $M$ is umbilical with respect to $F X$. So, by virtue of Theorem 3.1 it follows that $P X$ is conformal vector field. 
Theorem 3.7. Let $M$ be a submanifold of $\bar{M}$ with a concurrent vector field $X$ such that $\xi$ is normal to $M$. Then $(g, P X, \sigma)$ is a Ricci soliton on $M$ if and only if the following condition holds:

$$
S(Y, Z)=-\sigma g(Y, Z)-g(h(Y, Z), F X)
$$

for any $Y, Z$ tangent to $M$.

Proof. Using (3.5) in (1.5), we get the equation (3.14).

Theorem 3.8. Let $M$ be a submanifold of $\bar{M}$ with a concurrent vector field $X$ such that $\xi$ is normal to $M$ and $(g, P X, \sigma)$ is a Ricci soliton on $M$. Then $P X$ is conformal if and only if $M$ is umbilical.

Proof. Since $(g, P X, \sigma)$ is a Ricci soliton on $M$, then we have (3.14). Also since $P X$ is conformal, using (3.7) in (1.5) we have

$$
S(Y, Z)=-\sigma g(Y, Z)-\mu g(Y, Z)
$$

From (3.14) and (3.15) we can say that $M$ is umbilical.

Conversely, suppose $M$ is umbilical. Then we have the equation (2.9). Using (2.9) in (3.14) we get

$$
S(Y, Z)=-\sigma g(Y, Z)-\mu g(Y, Z) .
$$

Using (3.16) in (1.5), we obtain

$$
\left(\mathcal{L}_{P X} g\right)(Y, Z)=2 \mu g(Y, Z)
$$

which means that $P X$ is conformal.

Acknowledgement. The authors are thankful to the referee for her/his valuable suggestions towards to the improvement of the paper.

\section{References}

[1] D. E. Blair, Contact manifolds in Riemannian geometry, Lecture Notes in Math. 509, SpringerVerlag, 1976.

[2] B.-Y. Chen, Some results on concircular vector fields and their applications to Ricci solitons, Bulletin of the Korean Mathematical Society, 52(50) (2015), 1535-1547.

[3] B.-Y. Chen, Topics in differential geometry associated with position vector fields on Euclidean submanifolds, Arab J. Math. Sci., 23 (2017), 1-17. 
[4] B.-Y. Chen, Differential geometry of rectifying submanifolds, Int. Electron. J. Geom. 9 (2) (2016), 1-8, Addendum to 10 (1) (2017), 81-82.

[5] B.-Y. Chen, Euclidean submanifolds with incompressible canonical vector field, Serdica Math. J. 43 (3) (2017), 321-334.

[6] B.-Y. Chen, Harmonicity of 2-distance functions and incompressibility of canonical vector fields, Tamkang J. Math. 49 (2018), 339-347.

[7] B.-Y. Chen and S. Deshmukh, Classification of Ricci solitons on Euclidean hypersurfaces, Intern. J. Math. 25 (11) (2014), 1450104 (22 pages).

[8] B.-Y. Chen and S. Deshmukh, Ricci solitons and concurrent vector fields, Balkan J. Geom. Appl. 20(1) (2015), 14-25.

[9] B.-Y. Chen and S. Deshmukh, Yamabe and quasi-Yamabe solitons on Euclidean submanifolds, Mediterr. J. Math., 15 (2018), 194, doi.org/10.1007/s00009-018-1237-2.

[10] B.-Y. Chen and S. Deshmukh, Euclidean submanifolds with conformal canonical vector field, Bulletin of the Korean Mathematical Society, 55 (2018), 1823-1834.

[11] B.-Y. Chen and S. W. Wei, Differential geometry of concircular submanifolds of Euclidean spaces, Serdica Math. J., 43 (2017), 35-48.

[12] R. S. Hamilton, The Ricci flow on surfaces, Mathematics and general relativity, Contemp. Math., American Math. Soc., 71 (1988), 237-262.

[13] K. Yano and M. Kon, Structures on manifolds, World Sci. Publ. Co., Singapore, 1984.

Pradip Mandal Department of Mathematics, The University of Burdwan, Golapbag, Burdwan 713104, West Bengal, India

E-mail: pradip2621994@rediffmail.com

Yadab Chandra Mandal Department of Mathematics, The University of Burdwan, Golapbag, Burdwan 713104, West Bengal, India

E-mail: myadab436@gmail.com

Shyamal Kumar Hui Department of Mathematics, The University of Burdwan, Golapbag, Burdwan 713104, West Bengal, India

E-mail: skhui@math.buruniv.ac.in 